doi: $10.31211 /$ interacoes.n34.2018.a5

\title{
Media Shy: The Perils of Bashfulness in the Digital Age, an Era of Speed, Satisfaction, and Spectacle
}

\author{
Natasha Chuk \\ School of Visual Arts, New York - nchuk@sva.edu
}

\begin{abstract}
Speed and satisfaction are central to today's rithmic design and the commodification of digital communication tools and online social users. An examination of the history of online environments. This article examines how new forms and habits of social communication in digital environments have over time compromised socially timid users as a result of algosocial environments and their outgrowth, the consideration of social and cultural factors, and self-presentation theory, will be used to frame these arguments.
\end{abstract}

Keywords: Identity, medium theory, self-expression, self-presentation, social media

\section{Timidez nos Media: Os Perigos do Acanhamento na Era Digital, uma Era de Velocidade, Satisfação e Espetáculo}

\section{Sumário}

A velocidade e a satisfação são fundamentais para as ferramentas de comunicação digital e ambientes sociais on-line de hoje. Este artigo examina como as novas formas e hábitos de comunicação social em ambientes digitais comprometeram, ao longo do tempo, utilizadores socialmente tímidos como resultado do design algorítmico e da mercantilização dos utilizadores. Um exame da história dos ambientes sociais on-line e seu desenvolvimento, a consideração de fatores sociais e culturais e a teoria da auto-representação serão usados para enquadrar esses argumentos.

Palavras-Chave: Identidade, teoria dos média, auto-expressão, auto-representação, média sociais

'Oh, they don't miss me,' she said. 'I'm antisocial, they say. I don't mix. It's so strange. I'm very social indeed. It all depends on what you mean by social, doesn't it? Social to me means talking to you about things like this' - Fahrenheit 451 (Bradbury, 1953, p. 29). 
The benefits and advantages of digital media technologies and specifically networked connectivity and engagement are clear: speed, efficiency, and the prospect of doing more with little or no effort are appealing attributes and ways of life, irreversibly changing the ways we buy, sell, educate, learn, and share. However, as we proceed with using these tools with greater speed and arguably less contemplation, we run the risk of automating our behaviors and interactions based on the tendencies or biases of the mechanisms themselves. Perhaps most significant are the ways that socialization, self-expression, and individuality have shifted, allowing new perceptions and ways of being to emerge. New ways of cultivating personal and collective identities and guiding self-expression have been a boon, whereas the disadvantages are much more difficult to calculate or understand. The downsides of our digital world are numerous, though less readily communicated and more nuanced and tedious to gauge, in part because the flaws feel like reasonable tradeoffs for the benefits we enjoy and are unwilling, at this point, to relinquish. However, the aim of this article is to shed light on a particularly invisible disadvantageous group of users and address an issue of inequality that has gone relatively unnoticed but speaks to the ways that new media technologies inadvertently exclude certain groups of people in specifically social contexts to an extent that such groups have little or no recourse. More specifically, this article will focus on how many uses of networked connectivity, particularly but not limited to social media, can be detrimental to users who struggle with shyness and social vulnerability. This is achieved through an examination of the persuasive power of media by design and the commodification of users, which creates preferred or dominant uses that limit or complicate self-expression for this unique group, as well as through a theoretical framework for why and how such media are shaped by and in turn shape these outcomes. This article also addresses what constitutes shyness and how dominant communications technologies shape users through comparisons to other media. Medium theory and aspects of representation, self-expression, social constructs and their effects will be used to frame these arguments.

\section{BIAS AS A PRECONDITION TO ALL MEDIA}

One of the most fascinating realizations about media is that they are inherently biased toward various outcomes based on the qualities and character of their attributes. Medium theory holds that the form of a message - the features, and indeed the 
Media Shy: The Perils of Bashfulness in the Digital Age, an Era of Speed, Satisfaction, and Spectacle

structure, that make up the channel through which a message is transferred - is as important to understand its meaning and significance as the content itself. Communication theorist Harold Innis situated his studies within this theoretical framework, writing, 'a medium of communication has an important influence on the dissemination of knowledge over space and over time and it becomes necessary to study its characteristics in order to appraise its influence in its cultural setting' (1951/2008, p. 33). His observations primarily concern the material components of a medium, such as its weight, durability, or mobility. For example, 'writing on clay and on stone has been preserved more effectively than on papyrus' (Innis, 1951/2008, p. 33), which, for Innis, makes these durable media biased toward time: stone and clay will inevitably outlast papyrus. Additionally, we could add that a newspaper, though short-lived by comparison, is biased toward space: its lightweight and inexpensive qualities make it accessible to a wider audience, and therefore its larger reach increases its impact among users. While Innis's theories center on the transfer of knowledge, what we can take from that term is a more general and thus more sizable understanding of what might be more accurately referred to as simply information: alphabetic text, numeric text, moving images, still images, and so on. His findings confirm that a medium's characteristics play a sizable role in shaping meaning and the ways one might interpret the information being transferred. Moreover, users transform their habits, values, ideas, and identities based on the types of media that define their era. He continues, 'immediately we venture on this inquiry we are compelled to recognize the bias of the period in which we work' (1951/2008, p. 33). Neil Postman later echoed this sentiment, stating, 'ff course, like the brain itself, technology has an inherent bias. It has within its physical form a predisposition toward being used in certain ways and not others' (1985, p. 84). Naturally, the ways in which media are used affect the types of users using them.

The dominant medium today is the Internet and, more specifically, channels of social media, such as Facebook, Instagram, YouTube, Twitter, and so on. The biases toward lasting power and reach have been combined within their exchanges, and while this may seem somewhat inconsequential, especially as our modern world hosts numerous types of endurable media within and outside of networked environments, these biases are influential in themselves, regardless of the medium's content, and are default attributes to the media that dominate the present era. The crucial aspect of Innis's theory is the consideration of how information is disseminated within societies 
and how those exchanges transform them. Today, information is primarily exchanged via networks that promote shareability and exchange through specific attributes of design and programming. The immaterial nature of the digital form has ushered an entirely unprecedented bias of manipulation and control that favors certain users' behaviors and interactions over others in discreet but powerful ways. One noticeable shift is the desire for speed and convenience as two driving forces behind not only digital media design but also the ways that users engage both media and content. Google, the world's largest internet search engine, is an example of the downsides of designing for speed and convenience or relevance. With its gargantuan, unbreakable hold on users, especially in western societies where it originated, it operates through a complicated (and publicly undisclosed) calculated system of information retrieval, whose highly classified algorithm favors speed and advertising revenue over veracity and equality. Many have reported on algorithmic bias, for example, and the influence that large internet platforms like Facebook and Google had on the 2016 presidential election in the United States, during which they allowed the dissemination of targeted and incendiary messages to circulate for the purpose of swaying public opinion on various issues. 'For the makers of algorithms, the term refers specifically to the logical series of steps for organizing and acting on a body of data to quickly achieve a desired outcome' (Gillespie, 2016, p. 19). These programmed commands enact underlying values of the system: rapid information retrieval is one, and perhaps a second is popularity or relevance of content measured by trends and potential favorability.

The persuasive power of algorithmic design mainly lies in its surreptitious management of information: it is embedded in our systems - Google and beyond - and thereby hidden. An additional emergent bias is automation which, according to Lev Manovich, allows for a situation in which human interactions can 'intentionally be removed from the creative process, at least in part' (2001, p. 53). However, it is worth adding that automation also creates a template or model for human behaviors, which can serve as a creative substitute in the interest of convenience even when human agency is left intact and, as in the example previously mentioned, can dissuade users from critically thinking about how information is disseminated: automation discourages contemplation. 
Media Shy: The Perils of Bashfulness in the Digital Age, an Era of Speed, Satisfaction, and Spectacle

\section{AN EMERGENT BIAS TOWARD SHARING}

Sharing is the basis of the internet and is 'an important digital keyword not only because of its roots in computing (time sharing, disk sharing, file sharing, etc.), but because it bears the promise that today's network and mobile technologies - because they make it easier for us and encourage us to share extensively - will bring about a better society' (John, 2016, p. 270). The origins of digital society and its subsequent ideological values emerged within ARPANET (Advanced Research Projects Agency Network), the precursor to the World Wide Web, which formed in 1968/69 and set the stage for the web communications dynamic we presently utilize. There was evidence from its infancy of the great potential to not only conduct basic communication but also to build a virtual environment that promoted deep social ties among complete strangers. Digital networks have the unique advantage of allowing (semi) anonymity among users, helping alleviate superficialities and social barriers associated with the physical world, advancing a new understanding and practice of connection between actors, and in fact, creating an advantage for anyone inept at easily interacting with others in the physical social sphere. In person-to-person social settings we customarily evaluate one another based on appearance, vocal inflection, clothing and hairstyle, and more culturally nuanced attributes, like whether one is quick-witted and gregarious. By contrast, the first online communities of the 1990s gathered in chat rooms through services like America Online and CompuServe, and though it's unfair to idealize this time period and the kinds of services offered, at least their social aspects favored the user whose ideas could be 'socialized' merely through type without the social pressures of a visual or audiovisual performance in person. Media theorist Dana Boyd echoes this sentiment:

'When I embraced the internet as a teenager in the mid-1990s, I was going online to escape the so-called real world. I felt ostracized and misunderstood at school, but online I could portray myself as the person that I wanted to be. I took on fictitious identities in an effort to figure out who I was. I wasn't alone. Part of what made chatting fun in those days was that it was impossible to know if others were all that they portrayed themselves to be' (2013, p. 38).

Initially, being online meant that you were judged based primarily on what you typed, as the systems only utilized text-based inputs. Images, videos, graphics, and 
links were not yet supported on the first chat systems, and it was also common for users to select handles, or usernames, that bore no resemblance to their personal names, allowing them to form new personae as desired. Without such personally distinguishing details like names and photographs, other characteristics that provided a connection to one's physical form - age, gender, race, and physical appearance - weren't factored into one's personality profile by default. This afforded a certain level of removal or dissociation from one's physical self, and the chance to selectively conceal and reveal information as fancied. Moreover, nervousness, discomfort, and awkwardness brought on by person-to-person interactions were abetted, or at least hidden, and therefore were invisible to recipients. In these chat rooms, certain pressures of the physical world could be momentarily suspended to offer a respite for the shy and socially awkward. It is worth adding that shy types could in fact flourish in these environments given the unique affordabilities of the medium, which have dramatically shifted in recent decades toward establishing a connection to one's personal and physical life and restoring some of the social expectations of physical socialization through images and other media.

Today's digital sphere, ushered by Web 2.0 - a kind of redesign of the original World Wide Web - highly depends on social media that places sociability, not merely connection, at the center of most all digital communication, initially as a means to democratize the internet. According to Alice E. Marwick, 'Web 2.0 celebrated the adoption of social technologies as a precursor to a better, freer society, and framed the collection and sharing of information as the bedrock of revolution' (2013, p. 22). Social media is a broad term that refers to 'a group of Internet-based applications that build on the ideological and technological foundations of Web 2.0, and that allow the creation and exchange of User Generated Content' (Kaplan \& Haenlein, 2010, p. 61). These user-friendly web platforms thrive on what has become known as an economy of sharing, a system that is dependent on the contributions of billions of users who have grown accustomed to sharing personal details, images, and ideas. A byproduct of sharing is the notion that social relevance is tied to lively activity and contributions by participants. Social media, though divided by various dominant global platforms like Facebook, Twitter, Snapchat, and Instagram, have in common an emphasis on communities defined by users, followers, and lively activity that is connected in explicit ways to users' physical forms and personal lives. Facebook, for example, was created for the purpose of virtually connecting physical communities: users know 
Media Shy: The Perils of Bashfulness in the Digital Age, an Era of Speed, Satisfaction, and Spectacle

or knew each other in person, and eventually a web of strangers linked by common friends emerges. While it is customary to follow strangers on these platforms, the assumption is that users' contributions are somehow connected to their personal and physical lives. For the most part, meeting in person is not the end goal and in most cases it is impossible.

In addition to text, each of these platforms supports still and moving images, audio, web links, and graphics. It is not only accepted but highly expected that users contribute with images of their friends, family, home environment, and themselves, hence the dawn and immense popularity of the selfie, a 'type of digital self-portrait [that] is taken with a mobile phone and characterized by its ubiquity' (Wendt, 2016, p. 7). Moreover, these platforms favor quantity: members of communities (groups, followers) and their contributions (posts, likes, shares) are measured metrically and displayed visually, making explicit a numerical system of acceptance or disapproval, which is also archived for all to see and use as a tool of social evaluation. Finally, this emergent public sphere is cloud-based and ever present and, in that sense, dominant and inescapable. In addition to individual profile accounts, users often create professional profiles as do businesses and celebrities, adding tremendous commercial value and expectations to this social sphere.

\section{SOCIAL PERFORMANCE AND SELF-PROMOTION}

The significance of the social strata between early web chat rooms and today's social media is deeply tied to self-presentation, the behaviors and attitudes we present to one another in public. Media introduce interesting opportunities both for 'performing' and for social relations to take shape. As Erica Pearson asserts, online 'the audience and the performer are disembodied and electronically re-embodied through signs they choose to represent themselves' (2009). However, one could argue that the choice to engage such signs of personal preference are in rapid decline as a dominant mode of discourse presented by the use of personal images, confessions, and other ties to the physical world and identity has formed within social media, disparaging most alternative modes of communication. It has become increasingly clear that the social construct of social media reinforces habits and precepts of the physical social space to which the digital space felt strikingly dissimilar at one time, making 
the online social space of the present a more convenient and placeless substitute for a physical one, and bringing with it the attitudes and social mores that burden the socially awkward. A number of factors contribute to them, not least the ways that we behave in social groups and how we present ourselves to others. Appraisal and upward mobility are essential aspects of socialization, and a mix of cynicism and sincerity, according to Erving Goffman, help foster a bit of harmless self-illusion (1956, pp. 12-13), thereby benefiting the social actor. However, social performance precedes the internet and exceeds media communication as well.

As Goffman notes, 'performance' should be understood as 'all the activity of an individual which occurs during a period marked by his continuous presence before a particular set of observers and which has some influence on the observers' (1956, p. 13). The observable components, what Goffman calls the 'front', include the setting, appearance, manners, clothing, and more of the individual (1956, pp. 13-19). The 'front' is composed of characteristics that are impossible or difficult to conceal in physical situations but are, to some extent, obscured or partially hidden through media. For example, the telephone discloses the sound and character of one's voice but conceals his/her appearance and physical surroundings, while the written letter cloaks all but one's written ideas and penmanship. Online, typed text replaces handwriting, and image-based communication largely replaces the use of our voices in interpersonal communications. With this in mind, it would seem that the development of various personae in such seemingly inventive spaces could free one from the constraints of social awkwardness since, returning to Pearson, 'the mediated nature of these spaces means that most information about the virtual self and its place in the network is given through deliberate construction of signs, linking back to this sense of online self-consciousness' (2009). Theoretically, the freedom and ability to essentially invent oneself within these social spaces is possible through careful selection of signs and omission of undesirable personal attributes. In this sense, online social space is a tabula rasa that fosters a sense of imagination and self-expression. However, in practice, mainstream social media spaces are designed with different outcomes in mind, thereby suppressing certain behaviors and uses.

While social presence, along with self-presentation, precedes online and exceeds media communications, it's a key factor in how we might understand social media engagements today and the users that falter within it. Social presence is the 'acoustic, 
Media Shy: The Perils of Bashfulness in the Digital Age, an Era of Speed, Satisfaction, and Spectacle

visual, and physical contact that can be achieved' between two social actors (Kaplan \& Haenlein, 2010, p. 61). A close idea to this is media richness, which is 'based on the assumption that the goal of any communication is the resolution of ambiguity and the reduction of uncertainty' (Kaplan \& Haenlein, 2010, p. 61). Social media are designed to promote a high degree of social presence and media richness, meaning a high degree of self-disclosure, in which one consciously or unconsciously reveals personal information that is consistent with the image one wishes to project (Kaplan \& Haenlein, 2010, p. 62). These media theories draw on Goffman's ideas about selfpresentation and performance: we desire to communicate thoroughly and accurately while presenting the finest version of ourselves to our publics. These appear to be optimal conditions for the shy person's dilemma, however what is missing from these observations are the downsides of social media engagement based largely on their design and subsequent influences on individual behavior resulting in the encouragement of self-promotion of individuals toward a kind of redirection of ideas.

Moreover, as Marwick argues, social media are 'predicated on the cultural logic of celebrity, according to which the highest value is given to mediation, visibility, and attention' (2013, p. 14). As in the physical world, users are influenced by one another and the kinds of messages and content posted, setting the tone, standards, and other various criteria for acceptable contributions, sometimes at the expense of sincerity. The desire to fit in and feel popular drives such behaviors, but the platforms themselves also encourage and discourage certain behaviors based on their designs' affordances and limitations. As Christian Fuchs observes, 'competitive social media foster the branding, quantification, marketization, commodification, capitalization of the self' (2017, p. 36). They do this by design: users accumulate likes, followers, commentary, shares, and other forms of publicly displayed, quantifiable attention, which promotes behaviors aimed at increasing one's status within these media channels, and thereby keeps users returning (or never leaving) the platform. The incentive to participate operates on multiple levels: users are encouraged, by design, to contribute repeatedly, but they are also encouraged by fellow users to continue contributing after experiencing an endorsement, further cementing their popularity. However, its important to note that information in these channels operates under a hidden algorithmic bias: the system rewards popular users and their content, and essentially punishes - through low-visibility or absence altogether - unpopular users and their content. 'Facebook notoriously curates the user's content for them while allowing only a modicum of 
configurability within the larger parameters of the platform' (Pettman, 2016, p. 79). Within this space, the content located in one's feed is the central driver of information and ultimately influences users within the social space and their opinions of other users. With this in mind, popularity and shyness have never been more explicitly linked than they are in social media exchanges. Additionally, the increasing concern is that we are becoming slaves to the hidden operations of the algorithms, allowing these biases to go deeper than the branding of the digital self, as stated previously, whereby a sensationalist tendency is being built into the systems themselves. 'Social media is inherently libidinal to the degree that we participate in it in order to be recognized, endorsed, verified, or, if one is more a voyeur than an exhibitionist, to find objects of cathexis and fetishism' (Pettman, 2016, p. 108).

Because algorithms favor lively activity, meaning the content that generates more likes and attention generates more generation and more attention, we inherently seek to succeed as social actors in this system by contributing potentially more enticing and likeable content. This recalls the influence of automation mentioned earlier in this article. While social media users hold a considerable amount of agency, patterns and trends inevitably emerge from these platforms as it becomes clear what kinds of posts gain the most popularity, require less time and effort to construct, and thus achieve (programmatically speaking) more visibility on a platform. Moreover, social media contributions by individual users also compete with contributions made by advertisers, celebrities, and other professionals, setting a social standard that dramatically deviates from the modes we learn to engage in the physical world among local communities and, more significantly, favoring content conducive to extroverted users. This leaves many introverted and self-conscious users bereft of ways to effectively socialize in the digital social sphere.

\section{THE SHY-BOLD SPECTRUM}

In his book 'Shrinking Violets: The Secret Life of Shyness', Joe Moran refers to the shy-bold continuum, a spectrum of behaviors that register somewhere between apprehensive and modest (shy) to audacious and heroic (bold) among animals. Animal behaviorists and zoologists use this to examine various traits along the spectrum to study how animals socially network, organize, reproduce, and survive. Moran sug- 


\section{Media Shy: The Perils of Bashfulness in the Digital Age, an Era of Speed, Satisfaction, and Spectacle}

gests there might be a spectrum among humans worth examining, though he admits our behaviors are too nuanced and our situations too varied to classify them in this reductive way. However, it seems possible to utilize this spectrum to evaluate the qualities of a particular medium with respect to how it affords and limits various behaviors and actions among users. Though shyness is difficult to define beyond the desire or tendency to withdraw from social situations, Moran says it can also 'amount to an undue interest in others, a desire for human connection that defeats itself through anxiety and uncertainty' (2016, p. 1). Despite the subjectivity of shyness and the ways we use communication tools, the affordances and limitations through the design of various media undoubtedly shape the ways they are used. Dominant methods inevitably arise, regardless of which groups find themselves stumbling to master them. While shyness is by no means synonymous with weakness, the key to success for the socially sheepish in today's online social sphere is a feeling of agency, which is dwindling in today's digital media design as content that communicates sheepishness, and with that often contemplation and thoughtful intention, is ignored while its bolder, faster, and more spectacular counterparts are rewarded.

Given how easy it can be to assign (especially new) communication technologies a sine qua non designation without much consideration of merit or potential drawbacks, it is imperative that we examine these forms of persuasion in their affordances and limitations, which promote certain behaviors and applications over others. For the shy individual, media tools can both establish a haven for easy communication and a daymare of arduous delivery and lack of connection. As previously stated, media can shield certain personal attributes that would otherwise increase anxiety for the socially shy, but the design and popular uses of today's media favors the sharing of personal images and private details regarding daily life, making it difficult and at times socially unacceptable to deliver more nuanced or differently engaged messages. When one is too shy to articulate one's feelings publicly, even semi-publicly, social comfort is often found in less popular or secondary media, because under the right conditions, the socially timid can find ways both to express themselves and effectively fraternize, as discussed earlier with regard to chat rooms.

With this in mind, it seems that media have their own shy-bold continuum. For example, a published book will reach a certain public that includes friends, family, acquaintances, and strangers. Likewise, a tweet will reach one's followers and has the 
potential to be retweeted, screenshotted, or otherwise disseminated to those outside one's social network. In many ways the published book and the tweet are similar -both placing the work in front of friends and strangers and experiencing some kind of longevity -- but the former can be said to relieve shyness while the latter heightens it. The printed word, more definitive in its ability to withstand the test of time and distance, can feel less aggressive than a tweet, even though, with few exceptions, the latter will get buried in the continuous stream of incoming messages after a day or so given the volume of content Twitter users generate. It hasn't always been this way, but in the digital age, the printed word allows for a kind of delayed boldness, should there be any. The message can be high-impact, but its effects are comparatively incremental and cumulative. Physically printed messages require more time, effort, and reflection to decipher and react to. The message in a tweet, on the other hand, can seem to be more high-impact than it is, simply based on its means of delivery, which is one that is conducive to immediate response and a penchant for novelty, speed, and spectacle. Each form attracts users across the shy-bold continuum for different reasons, but the former can be said to have medium properties that favor the apprehensive and modest, while the latter can be said to indulge the whims of more courageous users. The latter is a shout while the former is more of a whisper or gentle nudge: both are in their own ways effective modes of self-expression, but the book is increasingly overlooked and deemed irrelevant to be as socially effective compared with the tweet.

Much of this has to do with the desire for convenience and efficiency. The tweet and other content located within streams are intended to be brief and succinct, while the longer formats - articles, books - require more time and attention to consume and decipher. That our attention spans have shortened over time is a criticism that has been debated, but there is evidence to suggest not that we have lost the ability to spend quality time and attention on various messages but that our tools do not require it and therefore urge us not to. 'When our responses are preempted and shaped by the protocols of the network - "only 140 characters... only 'like' button... only memes or emojis accepted" - then our critical faculties start to follow the same subroutines of the algorithm' (Pettman, 2016, pp. 120-121). Contemplative, unique responses are discouraged and uniformity and repetition sets in, which damages the potential for creativity and self-expression, as well as sets a standard of social discourse that edges out any outliers among its user base. The introspective approach does not fit into this social scheme, and the online social world begins to take shape 
Media Shy: The Perils of Bashfulness in the Digital Age, an Era of Speed, Satisfaction, and Spectacle

through predictable fragments. It is a different way of thinking about Innis' theory about media's bias toward time: today's media are biased toward the amount of time required to send, receive, and decipher a message rather than the amount of time the message will endure.

\section{SELF-EXPRESSION IN THE DIGITAL AGE}

However, the question of time is not the only factor to consider: a rising interest in imagery, particularly as a means of self-expression, increases with these platforms. Presently, the biggest and most consistent trend on social media is the creation and dissemination of selfies. A modification of its predecessor, the self-portrait, the selfie assumes an attitude of informality and experimentation rather than contemplation and singularity. 'It was perhaps unforeseen that people would generate an astronomical amount of selfies and that our desire to capture and share selfies would become a global phenomenon' (Wendt, 2016, p. 7). The reasons for it are unquestionably tied to the fascination with and wonder of the self, a human trait that dates back centuries through experiments with glass and metallurgy that produced the first reflective surfaces and thus the first images of the self. The self-portrait took this contemplation further to produce objects, mobile versions of our likeness, in paint and eventually photography. The modern subject is a by-product of these experiments and practices, as we continue to look inward to question, study, and enjoy. The selfie is an extension of this, in many ways engaging the philosophy and stance of the first users of chat rooms and the way they valued their new online social spaces: selfies are casual, imperfect, and repetitious. Users do not limit themselves to one, rather allow the selfie to be the locus of a particular message, a means of expressing one's thoughts or reflections at various times and situations. Because of their popularity and their emphasis on the individual user, the ongoing criticism is that selfies signal a sociological problem of rampant narcissism or other disorder of self-indulgence. However, they are undoubtedly a mode of discovery, self-expression, and a respite from daily life. Drawing on Marshall McLuhan, Wendt writes, 'by gazing upon our reflections, we receive momentary relief from stressful situations or personal anxieties - a break from reality. We are amplified by this process and therefore receive satisfaction from capturing and viewing selfies' (2016, p. 8). With this in mind, selfies underscore the values of the early web and its social domains, advancing performative opportunities and the enactment of various personae. However, 
because platforms like Instagram and Facebook encourage followers to like and comment on our posts, selfies are subject to the scrutiny of popularity and qualities of social acceptance. As Wendt observes,

As we tilt, raise, and lower our smartphones to find the best angle of ourselves on screens, we build perceptions about ourselves that are constructed purely from within screens. Instagram, thus, is not just a way to produce images but it is also an active means for some people to establish their identities - viewing the ubiquity of their selfies as a mark of distinction $(2016$, p. 7).

Distinction is an interesting term here when, in reality, selfies are deeply coded as best practices have emerged as guideposts toward ensuring successful, meaning socially acceptable, image captures. Wendt continues, noting how 'one can witness the power of this spell upon society in several YouTube tutorial videos titled "How to Take a Selfie"' (2016, p. 8). It isn't new that a formula has developed around how to take selfies (and profile pictures), but the scale and conditions around which selfies have become and remain popular make them an important means of socializing and demonstrating not so much individuality and self-expression but community and belonging.

Another obvious downside to selfies as a means of expression for the debilitatingly shy is their content: selfies are displays of the visible self. Regardless of filters, editing software, and other forms of manipulation, they are inherently troublesome for diffident users. It seems that this argument may go against McLuhan's idea that the medium is more considerable of a culture than the content of the message 'because it is the medium that shapes and controls the scale and form of human association and action' (1964/1994, p. 9). However, with regard to selfies and social media, the selfies are the message, meaning the selfies are a kind of micro medium. That this form of content has its own label is one reason to treat selfies as a sub-medium. In that sense, one can borrow and slightly adjust McLuhan's argument to better understand the impact of the selfie. He writes: 'In terms of the ways in which the machine altered our relations to one another and to ourselves, it mattered not in the least whether it turned out cornflakes or Cadillacs' (McLuhan, 1964/1994, pp. 7-8). Likewise, the message is the selfie itself, not the individual who appears as content, or the context in which the individual appears. 
Media Shy: The Perils of Bashfulness in the Digital Age, an Era of Speed, Satisfaction, and Spectacle

But there is another aspect of selfie taking that gives the reflective individual pause. One criticism was made by Peter Conrad, who lamented,

\begin{abstract}
The selfie is the perfection of solipsism: we assume that the rest of the world will share our high opinion of ourselves, and don't allow for the possibility that we might be viewed more sceptically. Obsessed with who we are, we forget where we are; rapt in self-consciousness, we squander our chance of attaining selfawareness (2016 p. 106).
\end{abstract}

This comment advances the idea that inveterate selfie takers are self-absorbed, but so much so that they fail to fully achieve self-awareness. According to this argument, the act grossly misses the potential and purpose of intentional play, experimentation, and personae-making. In fact, it concerns a more serious problem of mindless habituality and synchronization. Like the machines to which McLuhan refers, there is a kind of automation that is at work within the social media landscape. Cornflakes, Cadillacs, and other banal and worthwhile content alike stream through each user's feed. Regardless of content, the message is the stream and, oftentimes, the selfie. To socialize in this realm, one must add a contribution to the stream, like making an announcement in a crowded room. Unlike a crowded room, however, when no one hears your voice, that oversight is not publicized. Social media platforms display this information, noting just how many followers endorse your message, which requires more than simply hearing/seeing it. With this in mind, the selfie is the dominant mode not necessarily of self-expression but of self-presentation. It is a mode of digital presence, the baseline upon which other evaluations are made. Omitting this starting point, as a shy person might want to do, is like not showing up at the party at all.

Though there are differences among social media platforms, they each determine lively activity through engagement and reach, which allow and confirm that a user has enacted sociability. It is the platform's way of materializing sociality, to give it a form, but it is also a means to quantify it, as each platform's counter indicates. The number of endorsements (likes, shares, etc.) is visibly published for this very reason, creating a kind of popularity forum or, more crudely, a stage upon which one performs to encourage popularity. This aspect of social media is indicative of a significant shift away from the quality and character of the content, and toward an emphasis on the medium itself. Such behaviors and characteristics support conditions condu- 
cive to spectacle and the commodification of users rather than merely demonstrate self-expression. There is a sense that participants are engaged in acts of self-branding, extending the nature of performativity in everyday life described by Goffman. Individuals are quasi products in a system that advocates the promotion of one's appearance, opinions, status updates, and so on. Public endorsements and social follower counters are features that are unique to this environment, and there is no learned protocol for how to manage them except to attempt to reach higher numbers. This follows a business model rather than a human social one, turning our social spheres into popularity contests for the best products, or most effective users. McLuhan's assembly line metaphor returns as we adopt machine-like actions to produce ever more content: the message is the assembly line, not the content itself.

\section{OBSERVATION AND THE COLLAPSE OF PRIVATE AND PUBLIC SPACE}

The internet, which is for many the dominant social sphere of interaction, confuses private and public space. As José van Dijck observes, 'today, this layer of platforms influences human interaction on an individual and community level, while the worlds of online and offline are increasingly interpenetrating' (2013, p. 4). Fuchs adds, 'the boundaries between public life and private life as well as the work place and the home have become porous (2017, p. 50). Using the glass bedroom as a metaphor, in which a once private space becomes somewhat or entirely public, Pearson says our online social spaces require 'willing and engaged participation in mediated exchanges' (Pearson, 2009). As in the physical world, we observe each others behaviors in social exchanges. Online, those exchanges are mediated, and therefore abstracted and archivable. The abstracted aspect is critical, because it means the coded information is drawn from its physical-social counterpart but is nonetheless a fragmented interpretation of it and therefore demonstrative of a system that in many ways operates independently of our in-person social exchanges. This could be beneficial to users. As Dana Boyd observes among teens, 'paradoxically, the networked publics they inhabit allow them a measure of privacy and autonomy that is not possible at home where parents and siblings are often listening in' (Boyd, 2014, p. 19). The same, perhaps, could be said about letter writing or in some cases telephone use. Mediated social exchanges can sometimes allow privacy from physical publics. That information exchanged through social media platforms is archived (creating a bias toward 
Media Shy: The Perils of Bashfulness in the Digital Age, an Era of Speed, Satisfaction, and Spectacle

duration), even momentarily, but almost always exchanged in real-time (creating a bias toward immediacy) also is significant: one can refer back to a message at another time or context, imbuing it with potentially new meaning, which can be ignorant of the temporal space in which it was articulated. Compared to letter writing, which also allows one to write one's thoughts and creates the opportunity for duration and contemplation at a later time and place, the intended audience is often limited to one recipient, rather than a network (public) of friends, peers, and strangers, and the conditions in which the content of a letter is created, in most cases, is not imparted with immediacy, which encourages informality and lowers the formal protocols that help guide social exchanges. Moran acknowledges that this happens in person as well, in situations that encourage so-called small talk, noting, 'the real problem comes with informality, in casual encounters when conversations are meant to form artlessly, as if out of thin air' $(2016$, p. 2). Social media is conducive to the flow of artlessly formed chatter because it encourages real-time exchange and rewards charm, humor, and fearlessness, traits that the socially shy long to possess. Mistakes can and are frequently made within this space of cursory social engagement, but the socially courageous respond effortlessly: like falling awkwardly in public, the socially timid retreat ever further into their shells after a social blunder. And in an age in which online exchanges are imperative, it is difficult to avoid the online social sphere altogether. Moreover, Pearson adds, 'whilst it is true that users can lurk, even watching a performance constitutes a form of engagement. Users who refuse to watch, refuse to perform themselves, as a result see their own bonds atrophy' (2009). Passively watching online mirrors the shy person's actions in a physical social setting: the wallflower is the loner who is present but not actively engaged. In person, this could be assuaged by a small gesture - eye contact, a laugh, or a smile. Online the same is true: the digital equivalents of such small physical gestures are the 'like' or 'share', but in the physical realm one's social acceptance is not quantified, shared, and archived. Both successes and failures are much more nuanced and intricate in daily interactions.

The biggest difference between physical and online sociality is, again, the algorithmic determination of what happens to the users deemed wallflowers. Social media platforms are algorithmically calculated to optimize for time spent on the platform, which increases advertising revenue. In a crowded stream of users, the popular posts are contributed by popular users. The algorithm recognizes that users with more followers will reap the rewards of more engagement, while users with smaller followings 
and less frequent contributions literally get lost in the stream, mathematically pushed aside. This caters to marketing logic that favors reach and user feedback, which creates an algorithmically, rather than organically, determined hierarchy within the social sphere. While Facebook has been making tweaks to its algorithm after heavy criticism about its bias toward popularity, the changes remain hidden from users, and besides, the strength of popular users has already been publicly established, making it difficult to challenge this trend in a meaningful way. Additionally, the established methods of posting, commenting, and sharing in particular ways are mimicked by users who witness the rewards (through data analytics) of successful behaviors.

We observe how others behave and how others respond to such behavior: this is true on- and off-line. Observation online, however, is particularly calculable not only through designed elements afforded by various platforms, as previously discussed, but also through mediation itself producing a kind of observer effect. Online, especially via social media channels, we make adjustments to our behaviors based on the visible (and quantifiable) effects we observe and each medium allows enough distance or removal to construct suitable, meaning socially acceptable, personae. The immediacy engendered by such interactions creates comparatively raw and unfiltered content, mimicking in-person conversations, but this content dramatically differs in its staged calls for approval: the measured endorsements, or lack thereof, require targetable action/inaction, well beyond an in-person gesture. While these mediated exchanges can, therefore, encourage the cultivation of multiple personae, they discourage certain kinds of behaviors. In other words, users are not free to enact shyness in these exchanges; shy users are free to be anything else.

\section{CONCLUSION}

It was only a matter of time before social activities would shift to the virtual space of timeless- and placenessness, ushering a new convenience for socializing and communal activity. We engage friends, family members, acquaintances, colleagues, and strangers in fragments, sporadic interactions involving images and short threads of text that theoretically could cushion one's social awkwardness but in fact exacerbate it. The unspoken expectation is one of social bravery and fortitude: for the most part, private is public and audaciousness is celebrated. The advantages among users are not 
Media Shy: The Perils of Bashfulness in the Digital Age, an Era of Speed, Satisfaction, and Spectacle

equal and, moreover, there is no formal training for how one may be fully and successfully socialized in these spaces, nor is there reasonable removal from them, given their virtuality and persistence, and therefore no form of relief, without intentionally removing oneself from the spaces altogether.

The socializations of these spaces, however, are unique and are not translatable from the person to person exchanges they attempt to imitate. As Allucquère Rosanne Stone notes 'social rules do not necessarily map across the interface between the physical and virtual worlds' (1995, p. 80). Content within virtual spaces competes for attention in a stream of images and text that populate their platforms. In this way, they favor brassy commentary and imagery that startles the senses, permitting the momentary disruption of the flow of content to achieve a successful outcome. Blog headlines, Facebook posts, Instagram images, and similar platforms are conducive to the ostentatious displays of communication. In this sense, they share the qualities and techniques of paid advertisements, creating a visual and tactical bleed between personal and professional posts in a continuous stream of content. But this general effect is not limited to social media. Video chat applications, like Skype and FaceTime, literally require one confronts others visually, but unlike person-to-person communication, videotelephony provokes social awkwardness through its latency of audio signals, a common problem among each of these platforms regardless of internet speed, grainy video, and irregularity among user set-ups. While these technical issues are merely inconveniences that do not reflect the users' abilities directly, they interfere with the progression of conversations and contribute to unnecessary misunderstandings, which are conditions that can be debilitating for the shy. These tools, in addition to images like selfies, literally show the user and require a demonstration of confidence and self-assurance. Additionally, one cannot ignore the observation aspect of our online social sphere, which is a more complex activity than merely seeing one another in real-time, and instead enables users to record, replay, and share such interactions. In this sense, unique behaviors are not meant to thrive in these mediated environments, rather we are encouraged to learn best practices in order to succeed within them.

Media tools can both ameliorate and exacerbate shyness or timidity among users. The parallels and intersections between media and shyness are revealed through the ways that the former engages the ability to conceal and reveal information with 
a sense of agency, and provide the opportunity for the user to perform accordingly. Some flexible features and customization of our tools, such as various privacy, visibility, and activity settings, can help give users a sense of control. However, if these options are hindered, based on preferred uses by a majority of the members of the group and the systems that impede alternative interactions, agency for the outsider is compromised and the options are rendered pointless. Awareness of these issues is key to all users, shy and bold alike, who have the agency to shift the social discourse away from the preferences toward highly sensationalized content users favor and the system encourages. With these important issues in mind, alternative uses and platforms can and should emerge.

\section{REFERENCES}

Boyd, Dana (2014). It's complicated: The social Lives of networked teens. New Haven, CT: Yale University Press.

Bradbury, Ray (1959). Fahrenheit 451. New York, NY: Ballantine Books.

Conrad, Peter (2016). Mythomania. New York, NY: Thames \& Hudson.

Fuchs, Christian (2017). Social media: A critical introduction. Thousand Oaks, CA: Sage Press.

Gillespie, Tarleton (2016). Algorithm. In B. Peters (Ed.) Digital Keywords (pp. 18-30). Princeton, NJ: Princeton University Press.

Goffman, E. (1959). The presentation of self in everyday life. New York, NY: Doubleday Anchor Books.

Innis, Harold (1951/2008). The bias of communication. Toronto, CA: University of Toronto Press.

John, Nicholas (2016). Sharing. B. Peters (Ed.) Digital Keywords (pp. 269-277). Princeton, NJ: Princeton University Press.

Kaplan, Andreas \& Haenlein, Michael (2010). Users of the world unite! The challenges and opportunities of Social Media. Business Horizons, 53, 59-68. 
Media Shy: The Perils of Bashfulness in the Digital Age, an Era of Speed, Satisfaction, and Spectacle

Manovich, Lev (2001). The language of new media. Cambridge, MA: MIT Press.

Marwick, Alice E. (2013). Status update: Celebrity, publicity, and branding in the social media age. New Haven, CT: Yale University Press.

McLuhan, Marshall (1964/1994). Understanding media. Cambridge, MA: MIT Press.

Moran, Joe (2016). Shrinking violets: The secret life of shyness. New Haven, CT: Yale University Press.

Pearson, Erika (2009). All the world wide web's a stage: The performance of identity in online social networks. First Monday, Volume 14, Number 3 - 2 March 2009. Retrieved from http://firstmonday.org/ojs/index.php/fm/article/view/2162/2127

Pettman, Dominic (2016). Infinite distraction. Cambridge, UK: Polity Press.

Postman, Neil (1985). Amusing ourselves to death: Public discourse in the age of show business. New York, NY: Penguin Books.

Stone, Allucquère Rosanne (1994). The war of desire and technology at the close of the mechanical age. Cambridge, MA: MIT Press.

Van Dijck, Jose (2013). The culture of connectivity: A critical history of social media. Oxford, UK: Oxford University Press.

Wendt, Brooke (2014). The allure of the selfie: Instagram and the new self-portrait. Amsterdam, NL: Institute of Network Cultures. 